\title{
Anterior implant placement in the presence of maxillary impacted teeth: Case reports
}

\author{
Da-Min Park ${ }^{1}$, Hyeong-Gi Kim ${ }^{1,2}$, Young-Kyun Kim ${ }^{1,3}$ \\ ${ }^{1}$ Department of Oral and Maxillofacial Surgery, Section of Dentistry, Seoul National University Bundang Hospital, Seongnam, \\ ${ }^{2}$ Office of Human Resources Development, Armed Forces Capital Hospital, Armed Forces Medical Command, Seongnam, \\ ${ }^{3}$ Department of Dentistry \& Dental Research Institute, School of dentistry, Seoul National University, Seoul, Korea
}

\begin{abstract}
Sometimes canine or supernumerary teeth are impacted at the premaxilla area in adult patients. If an impacted tooth exists at the implant site, the teeth are regularly extracted before implantation. On the other hand, it is unclear if the impacted tooth needs to be extracted for implantation. This paper presents the cases in which bone grafts were performed after tooth extraction and implants were placed and cases in which implants were placed without extraction. The aim was to provide answers to these questions along with a literature review. (JOURNAL OF DENTAL IMPLANT RESEARCH 2021;40(3):90-96)
\end{abstract}

Key Words: Impacted tooth, Mesiodens, Implant

\section{INTRODUCTION}

Supernumerary teeth are most common around the maxillary central incisors, and they appear mainly in an impacted state, with a prevalence of $0.1 \sim 3.8 \%{ }^{1)}$. In addition, impaction of the maxillary canine is the second most common after the maxillary third molar, with a prevalence of $1 \sim 2.5 \%$ reported $^{2-4)}$. Therefore, when implants are placed in the maxillary anterior region, impacted teeth may become an obstacle to implant treatment. Until now, extraction is usually performed when an impacted tooth exists in the area where the implant is to be placed $^{5}$. However, when implants are placed at the same time as the extraction of the impacted tooth, since severe bone defects exist, bone grafting is often required and it may be difficult to secure the primary stability of the implant. As the age increases, the impacted tooth is more likely to adhere to the surrounding bone tissue and the bone elasticity decreases, so surgical extraction can be very invasive $^{6}$. Also there is a problem in that the treat- ment period becomes very long if the impacted tooth is extracted and waiting for a natural recovery ${ }^{7}$.

Therefore, it is questionable whether implants should be placed after the impacted teeth have been extracted. On the other hand, if the implant is placed while leaving the impacted tooth, the question arises as to whether there is a problem with osseointegration if the implant comes into contact with or penetrates the impacted tooth.

Some previous studies have reported that there is no need to forcefully remove the implant when the residual root or impacted tooth remains and osseointegration can occur even if the implant penetrates or is placed close to the residual tooth, and no pathological change occurred between the implant and the residual tooth during long-term observation ${ }^{7-9)}$. In this paper, we present a case in which an implant was placed at the same time as a bone graft was performed after tooth extraction and a case in which an implant was placed with an impacted tooth left. Along with a literature review, we intend to present answers to these questions. This paper was writ-

Received July 20, 2021, Revised August 23, 2021, Accepted August 31, 2021. (c) Journal of Dental Implant Research.

This is an open access article distributed under the terms of the Creative Commons Attribution Non-Commercial License (http://creativecommons.org/licenses/by-nc/4.0) which permits unrestricted non-commercial use, distribution, and reproduction in any medium, provided the original work is properly cited.

Correspondence to: Young-Kyun Kim, https://orcid.org/0000-0002-7268-3870

Department of Oral and Maxillofacial Surgery, Section of Dentistry, Seoul National University Bundang Hospital, 82 Gumi-ro 173, 173beon-gil, Bundang-gu, Seongnam 13620, Korea. Tel: +82-31-787-7541, Fax: +82-31-787-4068, E-mail: Kyk0505@snubh.org 
ten under the approval of the Seoul National University Hospital Institutional Review Board (B-2108-701-701).

\section{CASE REPORTS}

\section{Case 1}

A 40-year-old female patient came to our hospital with the complaint of periapical lesion of \#63. The \#63 tooth was in need of extraction due to the severe bone loss, and the \#23 tooth was horizontally impacted (Fig. 1). Because the \#23 tooth was deeply impacted and required a lot of bone removal for access, we planned to place the implant immediately after extraction under general anesthesia, Tooth \#23 was extracted by approaching labially and after drilling according to the usual procedure, implant (Osstem TS III CA, 3.5D/13L, Osstem Implant Co., Busan, Korea) was placed in the \#63 extraction site. Primary stability was 62 Implant stability quotient (ISQ). The cover screw was connected and bone graft material (ExFuse, Hans biomed, Seoul, Korea) was implanted in bone defect area (Fig. 2). After 5 months, secondary surgery was performed, and secondary stability was 70 ISQ. The upper prosthesis was installed, and regular maintenance check

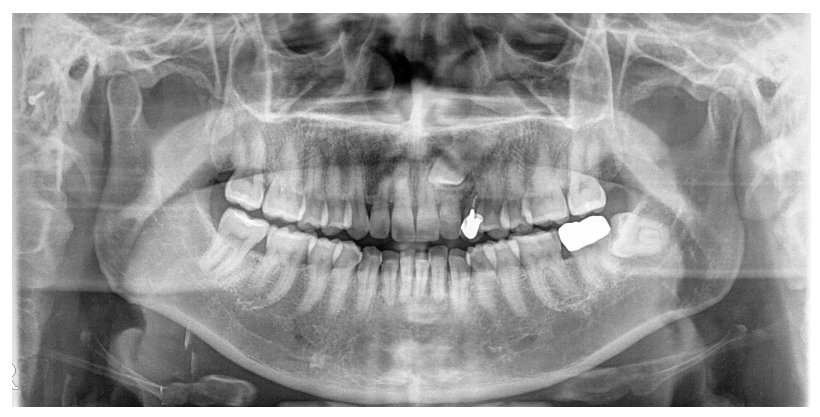

Fig. 1. Panoramic radiograph at first visit of a 40-year-old female patient. The \#23 tooth was horizontally impacted and the \#12 external root resorption was observed, but there was no mobility. was performed every 6 months. Two years after the first surgery, the patient complained that the sensation around the implant was different from that of the contralateral side. Other than that, there were no other abnormalities, and the implant remains stable after 5 years (Fig. 3).

\section{Case 2}

A 42-year-old male patient visited our hospital for implant treatment in the \#12 area. As a result of radiographs, it was confirmed that there were two supernumerary teeth around maxillary central incisor (Fig. 4). Since the supernumerary tooth was close to the nasal floor and there is a possibility of bony ankylosis, it was expected that severe bone loss would occur if extracted. Therefore, in order to avoid invasive surgery, it was planned to place the implant in a state where the impacted tooth was left unextracted. The patient consented after being informed of the treatment method and complications. The flap was raised after \#12 alveolar incision under local anesthesia, and implants (Osstem TS III CA,
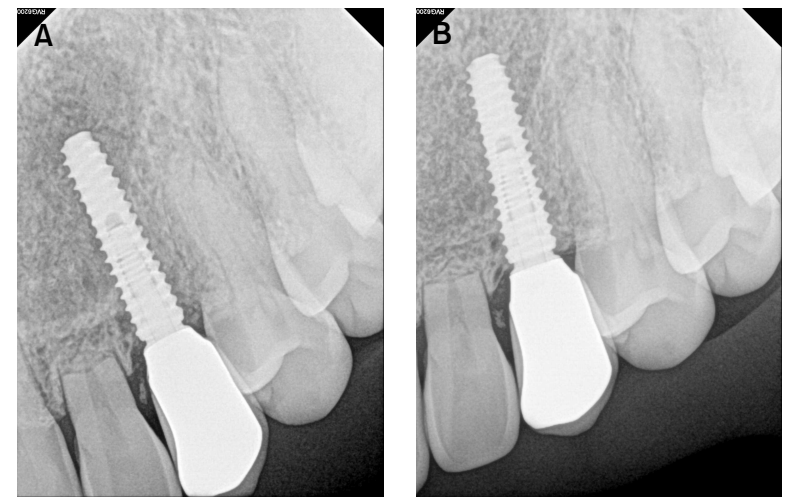

Fig. 3. Periapical radiograph after placement of the upper prosthesis. (A) Periapical radiograph right after prosthesis placement. (B) Periapical radiograph 5 years after prosthesis placement.
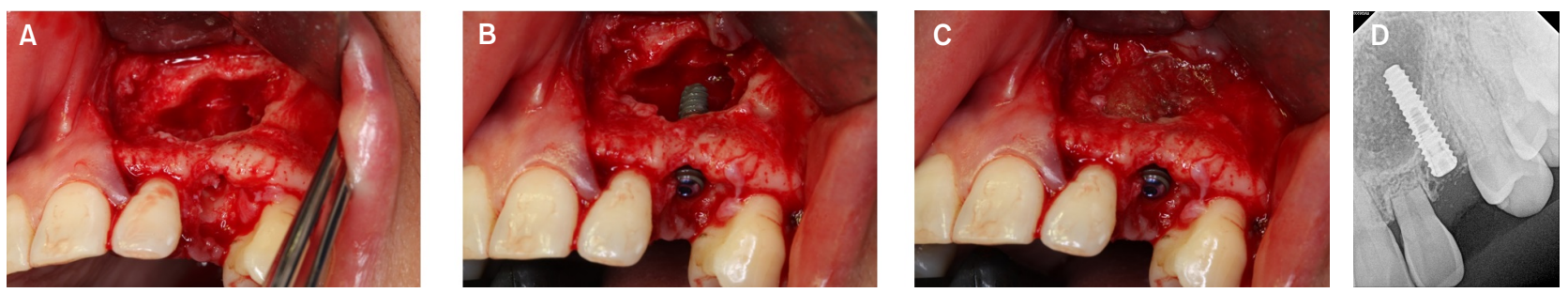

Fig. 2. Impacted \#23 tooth was extracted and the implant was placed immediately. Bone grafting was performed. (A) \#23 extracted. (B) Implant fixture placement. (C) Bone graft. (D) Periapical radiograph after implant placement. 

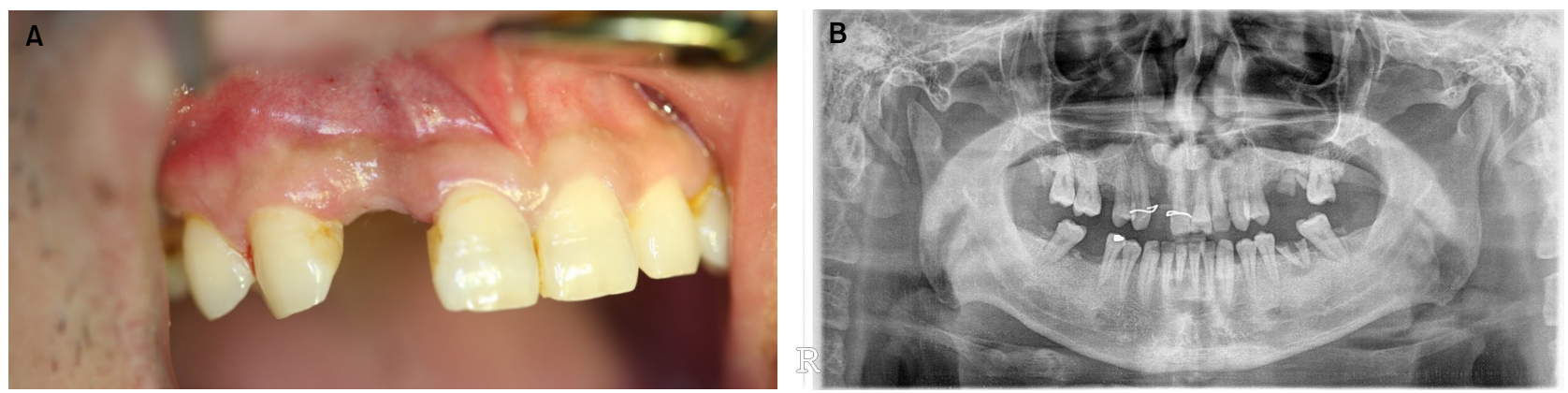

Fig. 4. At first visit of a 42-year-old male patient. \#12 were missing, and two supernumerary teeth were impacted around \#11 21 area near the nasal floor. (A) Intra-oral photo before surgery. (B) Panoramic radiograph.
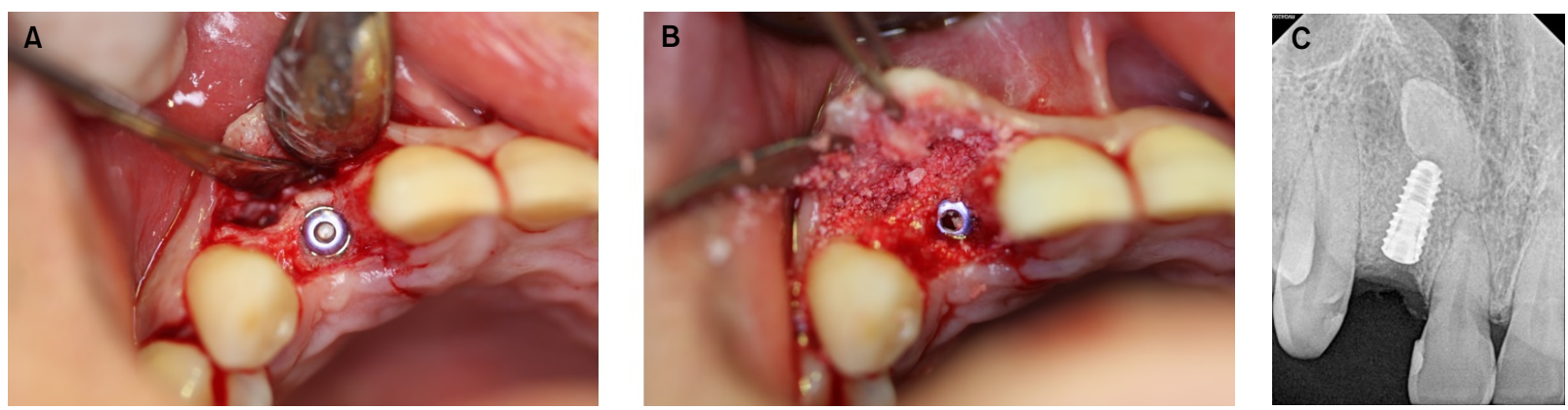

Fig. 5. Implants were placed with the impacted teeth remaining. (A) The implant was placed while expanding the alveolar bone using SplitMaster. (B) The cover screw was connected and bone graft was performed on the peripheral bone defect site. (C) Postoperative periapical radiograph present implant in contact with the root of the impacted tooth.

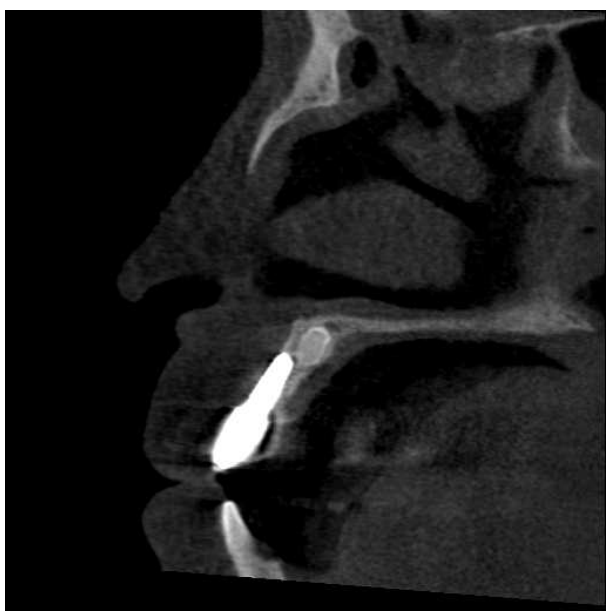

Fig. 6. Radiograph 1 month after placement of the upper prosthesis. On СBCT the root surface of the remaining supernumerary tooth and the implant fixture were in contact.

3.5D/8.5L) were placed while performing alveolar bone augmentation using Split Master (MCTBio, Yongin, Korea). After connecting the cover screw, bone graft (The Graft, Purgo biologics, Seongnam, Korea) was performed on the peripheral defect and the wound was sutured.

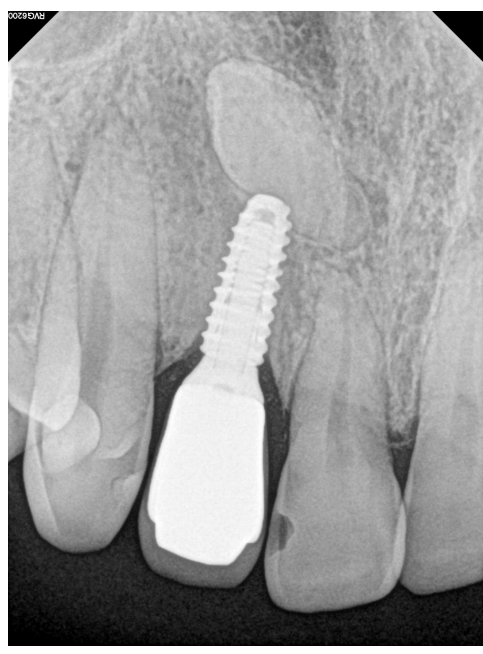

Fig. 7. 2 years after placement of the upper prosthesis.

Implant primary stability was 53 ISQ. The postoperative radiograph showed that the implant was in contact with the impacted tooth (Fig. 5). After 5 months, secondary surgery was performed, and secondary stability was 73 ISQ. Since then, the upper prosthesis has been installed and regular maintenance has been well performed for 2 

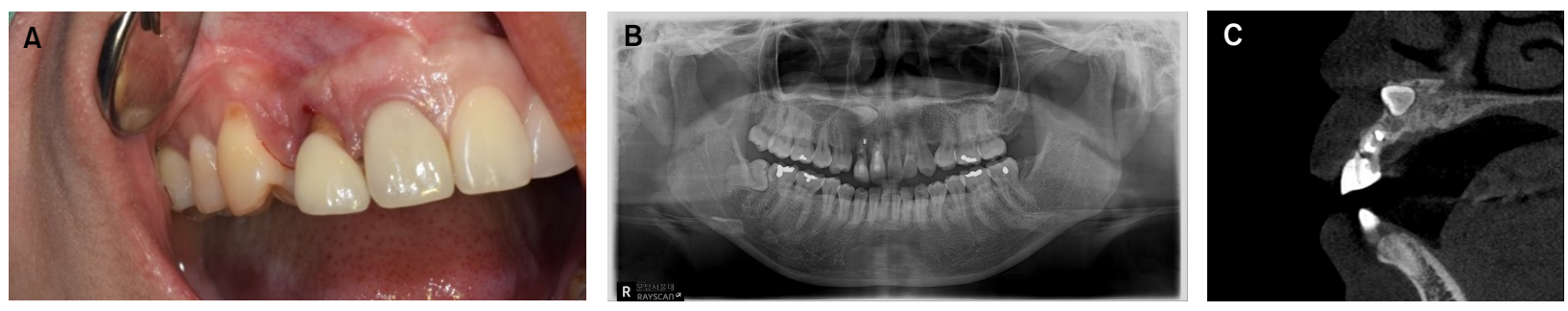

Fig. 8. At first visit of a 30-year-old female patient. (A) Intra-oral photo before surgery. (B) Panoramic radiograph. \#12 root fracture and horizontally impacted supernumerary tooth on the upper side. (C) СBCT. Short implant placement was planned with minimal impacted canine invasion.
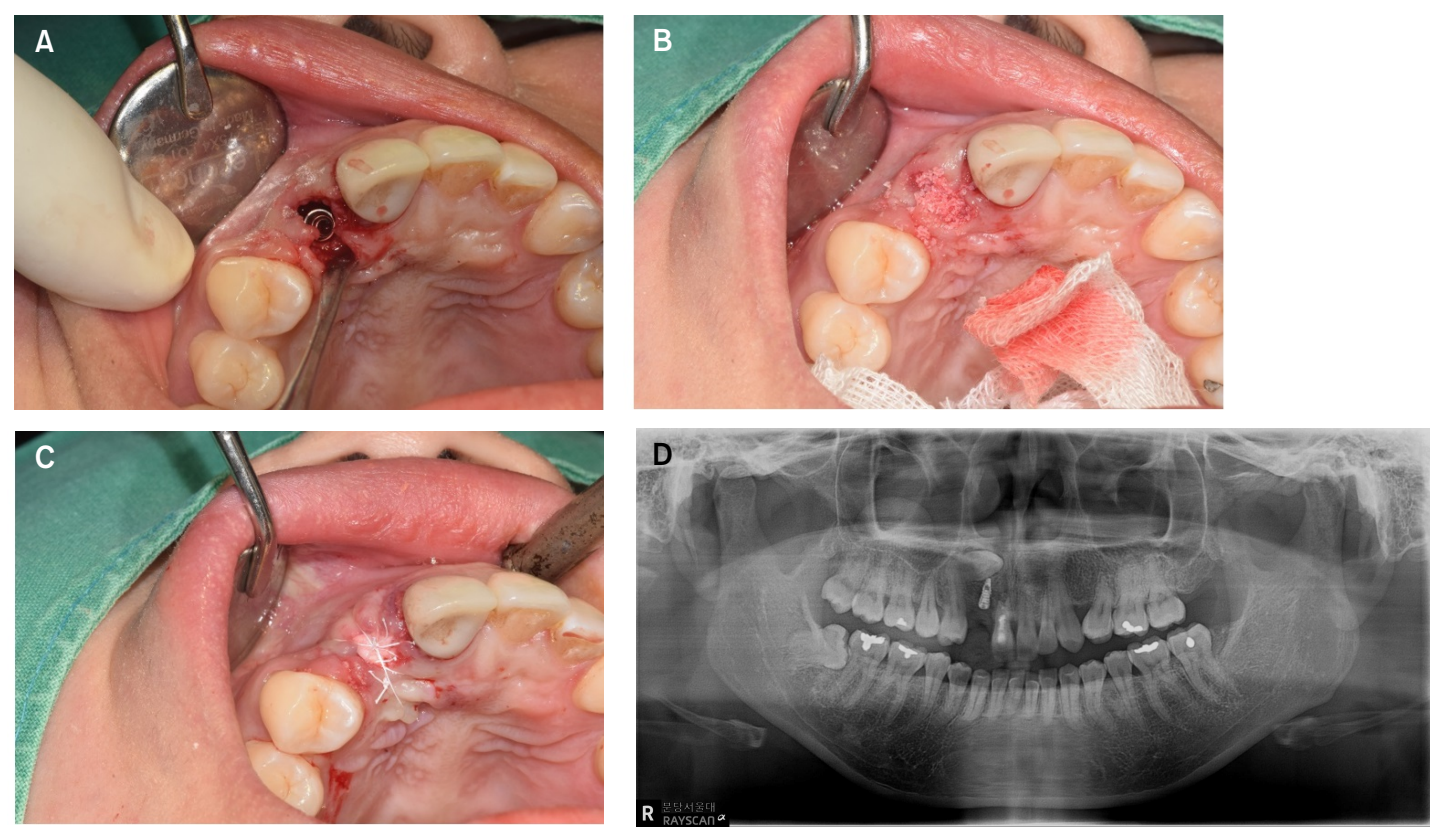

Fig. 9. Implants were placed immediately after extraction. (A) Implant (diameter $3.5 \mathrm{~mm}$, length $8.5 \mathrm{~mm}$ ) was placed close to the palatal side. (B) The Graft was transplanted into the surrounding space. (C) The Ossix membrane was covered and sutured (Open membrane technique). (D) Panoramic radiograph taken right after 1st surgery.

years after prosthetic loading (Fig. 6, 7).

\section{Case 3}

A 30-year-old female patient visited our hospital with \#12 tooth mobility, and was diagnosed with root fracture as a result of clinical and radiological examination. The canine was impacted between the \#12 root and the bottom of the nasal cavity, but it was decided to place an implant without invasive extraction of the impacted tooth (Fig. 8). After extraction of \#12, flapless drilling was performed on the palatal side, and a short implant (CMI 3.5D/8.5L, Neobiotec, Seoul, Korea) was placed (Fig. 9). Implant primary stability was 66 ISQ, and a cover screw was connected. A bone graft (The graft, Purgo biologics,
Seongnam, Korea) was performed in the surrounding space, covered with resorbable collagen membrane (OssixPlus, Dentsply sirona, Pennsylvania, USA), and sutured to maintain the barrier membrane well. After 6 months, secondary surgery was performed, secondary stability was 79 ISQ, and upper prosthetic treatment was performed. Since then, periodic maintenance care has been implemented, and the implant has been maintained well without any special problems for 2.5 years follow up (Fig. 10).

\section{DISCUSSION}

We have always had questions about whether im- 

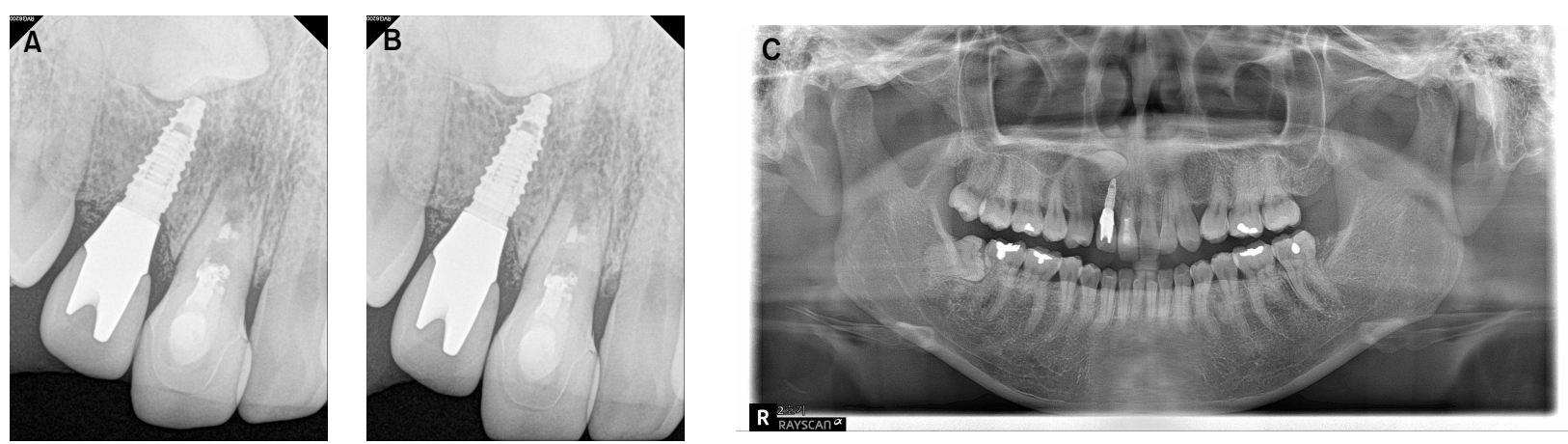

Fig. 10. Radiograph after placement of the upper prosthesis. (A) Periapical radiograph 1 year after prosthesis placement. (B) Periapical radiograph 2 year 6 months after prosthesis placement. (C) Panorama 2 year 6 months after prosthesis placement.

pacted supernumerary tooth extraction must be performed. Ferguson et al. noted that extraction is not essential if there are no symptoms or lesions associated with impacted teeth ${ }^{10)}$. In the presence of impacted teeth, problems such as permanent tooth eruption, tooth displacement, crowding, diastema, obstruction of permanent tooth root formation, cyst formation, and eruption into the nasal cavity may occur ${ }^{11-14)}$. Since most of these problems occur during tooth eruption, pathological changes such as cysts or tumors around impacted teeth should be considered in adult patients. It has been reported that dentigerous cysts related to maxillary impacted teeth account for about $5 \%$ of all dentigerous cysts ${ }^{15,16)}$. In addition, in a paper that followed 256 supernumerary teeth for a long time, it was reported that cystic changes were observed around only $11 \%$ of supernumerary teeth ${ }^{17}$.

There are other concerns about the possibility of osseointegration failure of the implant, pulp lesions of the remaining teeth, root resorption, and severe pain when an implant is placed in a nearby the impacted tooth remaining. According to a study by Amato et al., implants were placed close to 10 impacted teeth (7 at maxilla, 3 at mandible) and the success rate of implants was $100 \%$ after 5 to 7 years of observation ${ }^{7}$. According to an animal experiment by Warrer et al., when the implant is positioned adjacent to the periodontal ligament of the residual root, a periodontal ligament is formed in the empty space, and newly formed cement is accumulated on the surface of the implant where the residual root and the implant meet, then osseointegration occurs normally ${ }^{18)}$. Nik-Hussein et al. reported that even in long-term studies on implants placed adjacent to nor- mally functioning teeth (including cases of direct root invasion, root surface contact, and close contact with the root), most of the roots healed well naturally, so they noted that contact with the tooth is not simply a reason to remove the implant ${ }^{11)}$. Iqbal et al. noted that even if the implant invades the pulp, it rarely causes pathological changes or pain, and that pulp pain or necrosis is mainly caused by postoperative infection or nerve compression ${ }^{19)}$. In a recent study by $\mathrm{Yi}$ et al., after long-term follow-up of implants that invaded adjacent teeth for 7 years, there was little chance of clinical problems ${ }^{8}$.

On the other hand, some scholars mentioned that it is better to remove the implant when the residual root or foreign material is present at the implant site because it often causes lesions ${ }^{7,20)}$.

When placing implants in the presence of impacted teeth, it would be good to refer to the following guidelines presented by Amato et al. 1) If there is no pathologic lesion such as a cyst and the impacted tooth is healthy, it should remain. 2) The impacted tooth must be completely embedded into the bone (at least $5 \mathrm{~mm}$ of bone above the impacted tooth). 3) During implant placement, penetration is allowed only up to cementum and dentin. Care must be taken not to violate the dimensions. 4) There is no need to reduce the implant depth or width to avoid impacted teeth ${ }^{7}$.

In this paper, we dealt with the cases of three patients who had impacted teeth in the maxillary anterior region and needed implant placement in this region. In Case 1, the impacted tooth was extracted before implantation. The reason why we extracted it is as follows. The crown portion of the impacted tooth was located at the middle 
third of the root of the adjacent tooth, and about half of the root of adjacent tooth (\#22) was resorbed. Therefore, it was thought that if the implant was placed with the impacted tooth left, the placed implant could directly invade the pulp of the impacted tooth or cause additional root resorption of tooth \#22. The impacted tooth was surgically extracted and then the implant was placed immediately with a bone graft material.

In cases 2 and 3, implants were placed adjacent to the impacted teeth without extraction. The relationship between the impacted tooth and the placed implant in the postoperative radiographs is as follows. In case 2, the implant directly invaded the root, and in case 3, the implant was in contact with the periodontal ligament of the impacted tooth. Although both cases did not invade the pulp cavity, an implant of $3.5 \mathrm{~mm}$ in diameter and 8.5 $\mathrm{mm}$ in length was placed in order to minimize contact with the impacted tooth. In all of the cases, periodic maintenance care was well performed, and the implant maintained normal function.

The authors learned the following lessons and guidelines from these cases. If there is an impacted tooth where the implantation is planned, it should not be unconditionally extracted. If the conditions are suitable and the patient can be sufficiently explained, it is advisable to add the method of placing the implant in close proximity with the remaining teeth as one of the generally considered options.

When there is an impacted tooth around the area where implant placement is planned, it is necessary to reconsider whether unconditional extraction is the right treatment method. If the impacted tooth is located in the superficial layer or partially exposed, or if there are pathological findings such as inflammation, cyst or tumor, extraction is appropriate. However, it is necessary to consider the implant placement method using minimal invasive technique with the impacted teeth remaining in patients with systemic disease in the elderly whose tooth are deeply impacted and have ankylosis with surrounding bone. The potential problems such as osseointegration failure, infection, pain, and the possibility of re-operation should be well explained to the patient before the treatment procedure begins.

\section{CONCLUSIONS}

When there is an impacted tooth at the location where the implant is to be placed, it is recommended to consider placing the implant close to the impacted tooth rather than extracting it unconditionally.

\section{ORCID}

Da-Min Park, https://orcid.org/0000-0001-7319-4270

Hyeong-Gi Kim, https://orcid.org/0000-0003-4730-0396

Young-Kyun Kim, https://orcid.org/0000-0002-7268-3870

\section{REFERENCES}

1. Rajab LD, Hamdan MA. Supernumerary teeth: review of the literature and a survey of 152 cases. Int J Paediatr Dent 2002;12:244-54.

2. Pedro FL, Bandeca MC, Volpato LE, Marques AT, Borba AM, Musis CR, et al. Prevalence of impacted teeth in a Brazilian subpopulation. J Contemp Dent Pract 2014;15:209-13.

3. Cooke J, Wang HL. Canine impactions: incidence and management. Int J Periodontics Restorative Dent 2006;26:483-91.

4. Bishara SE. Impacted maxillary canines: a review. Am J Orthod Dentofacial Orthop 1992;101:159-71.

5. Demarosi F, Varoni E, Rimondini L, Carrassi A, Leghissa GC. Immediate Implant Placement After Removal of Maxillary Impacted Canine Teeth: A Technical Note. Int J Oral Maxillofac Implants 2016;31:191-4.

6. Becker A, Chaushu G, Chaushu S. Analysis of failure in the treatment of impacted maxillary canines. Am J Orthod Dentofacial Orthop 2010;137:743-54.

7. Amato F, Macca U, Amato G, Mirabella D. Immediate Loading of Implants Inserted Through Impacted Teeth in the Esthetic Area: A Series of 10 Cases with up to 7 Years of Follow-up. Int J Periodontics Restorative Dent 2019;39:325-32.

8. Yi YJ, Park IW, Ku JK, Jo DW, Han JS, Kim YK. Long term clinical result of implant induced injury on the adjacent tooth. Sci Rep 2021;11:7913.

9. Sohn JB, Kim YK. Implant Placed in Contact with Retained Root: Case Reports. Implantology 2016;20:142-50.

10. Ferguson JW. Management of the unerupted maxillary canine. Br Dent J 1990;169:11-7.

11. Nik-Hussein NN. Supernumerary teeth in the premaxillary region: its effects on the eruption and occlusion of the permanent incisors. Aust Orthod J 1990;11:247-50.

12. Foley J. Surgical removal of supernumerary teeth and the fate of incisor eruption. Eur J Paediatr Dent 2004;5:35-40.

13. Nam OH, Lee HS, Kim MS, Yun KH, Bang JB, Choi SC. Characteristics of Mesiodens and Its Related Complications. Pediatr Dent 2015;37:E105-9.

14. Colak H, Uzgur R, Tan E, Hamidi MM, Turkal M, Colak T. 
Investigation of prevalence and characteristics of mesiodens in a non-syndromic 11256 dental outpatients. Eur Rev Med Pharmacol Sci 2013;17:2684-9.

15. Vosough Hosseini S, Moradzadeh M, Lotfi M, Ala Aghbali A, Fattahi S. Dentigerous cyst associated with a mesiodens: a case report. J Dent Res Dent Clin Dent Prospects 2011;5:76-8.

16. von Arx T. Anterior maxillary supernumerary teeth: a clinical and radiographic study. Aust Dent J 1992;37:189-95.

17. Asaumi JI, Shibata Y, Yanagi Y, Hisatomi M, Matsuzaki H, Konouchi $\mathrm{H}$, et al. Radiographic examination of mesiodens and their associated complications. Dentomaxillofac Radiol
2004;33:125-7.

18. Warrer K, Karring T, Gotfredsen K. Periodontal ligament formation around different types of dental titanium implants. I. The self-tapping screw type implant system. J Periodontol 1993;64:29-34.

19. Iqbal M, Kim S, Yoon F. An investigation into differential diagnosis of pulp and periapical pain: a PennEndo database study. J Endod 2007;33:548-51.

20. Reiser GM, Nevins M. The implant periapical lesion: etiology, prevention, and treatment. Compend Contin Educ Dent 1995;16:768, 70, 72 passim. 